\title{
Search for Heavy Higgs Bosons Decaying into Two Tau Leptons with the ATLAS Detector using pp Collisions at $\sqrt{\mathrm{s}}=13 \mathrm{TeV}$
}

\author{
Hanfei $\mathrm{Ye}^{a, *}$, on behalf of the ATLAS collaboration \\ ${ }^{a}$ School of Physics, Nanjing University \\ Hankou Road22, Nanjing, China \\ E-mail: hanfei.ye@cern.ch
}

\begin{abstract}
A search for heavy neutral Higgs bosons is performed using the LHC Run 2 data, corresponding to an integrated luminosity of $139 \mathrm{fb}^{-1}$ of proton-proton collisions at $\sqrt{s}=13 \mathrm{TeV}$ recorded by the ATLAS detector. The heavy resonance is searched over the mass range $0.2-2.5 \mathrm{TeV}$ in the $\tau^{+} \tau^{-}$decay with at least one $\tau$-lepton decaying into handronic final states. The data are in good agreement with the Standard Model predictions. The observed (expected) upper limits are $1.8 \mathrm{fb}$ ( $3.8 \mathrm{fb}$ ) for gluon-gluon fusion and $1.1 \mathrm{fb}(2.2 \mathrm{fb}$ ) for b-associated production at the neutral Higgs boson mass of $1 \mathrm{TeV}$. Results are also interpreted in terms of several Minimum Supersymmetry Standard Model scenarios.
\end{abstract}

The European Physical Society Conference on High Energy Physics (EPS-HEP2021), 26-30 July 2021

Online conference, jointly organized by Universität Hamburg and the research center DESY

\footnotetext{
${ }^{*}$ Speaker
} 


\section{Introduction}

Many Beyond Standard Model (BSM) theories extend the Higgs sector of the Standard Model (SM) and predict additional Higgs bosons to address the phenomena not described by the SM. For example, the two-Higgs-doublet models (2HDMs) extend the Higgs sector and predict additional neutral and charged Higgs bosons [2]. One concrete realization of 2HDMs is the Minimum Supersymmetry Standard Model (MSSM) [2]. The Higgs sector of the MSSM contains two Higgs doublets, which results in five physical Higgs bosons after electroweak symmetry breaking. These five physical Higgs bosons include two neutral and CP-even (even charge parity) Higgs bosons ( $h$, $H$ ), one neutral and CP-odd Higgs boson $(A)$, and two charged Higgs bosons $\left(H^{ \pm}\right)$. At tree level of the MSSM, the Higgs sector is described by $m_{A}$, the mass of the CP-odd Higgs boson, and $\tan \beta$, the ratio of the vacuum expectation values of two Higgs doublets. The couplings of MSSM Higgs bosons to down-type fermions are enhanced with respect to the SM for large $\tan \beta$, which results in larger branching fractions to $\tau$-lepton and $b$-quark.

This report presents results of a search for neutral MSSM Higgs bosons in $\tau \tau$ final states using $139 \mathrm{fb}^{-1}$ proton-proton collision data collected by the ATLAS detector [1] in 2015-2018 at a center-of-mass energy of $13 \mathrm{TeV}$ [2]. Two major production modes of the neutral MSSM Higgs boson are considered in this search, the gluon-gluon fusion $(\mathrm{ggF})$ and the $b$-associated production $(b b H)$, as shown in Figure 1.

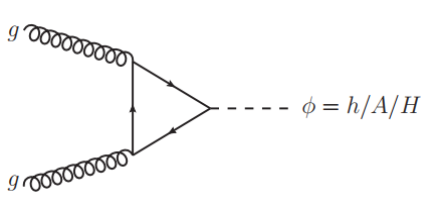

(a)

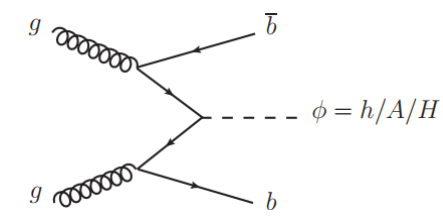

(b)

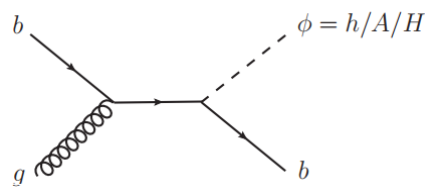

(c)

Figure 1: Feynman diagrams for (a) gluon-gluon fusion (ggF) and (b) b-associated production $(b b H)$ in four-flavour scheme and (c) five-flavour scheme of a neutral MSSM Higgs boson.

\section{Analysis strategy}

The analysis is split into two channels, $\tau_{\text {lep }} \tau_{\text {had }}$ and $\tau_{\text {had }} \tau_{\text {had }}$, where $\tau_{\text {lep }}$ means that a $\tau$-lepton decays into neutrinos and an electron $\left(\tau_{\mathrm{e}}\right)$ or neutrinos and a muon $\left(\tau_{\mu}\right)$ and $\tau_{\text {had }}$ denotes the decay of a $\tau$-lepton into a neutrino and hadrons. Events in the signal region are required to pass single lepton triggers in the $\tau_{\text {lep }} \tau_{\text {had }}$ channel, or to pass single $\tau$ triggers in the $\tau_{\text {had }} \tau_{\text {had }}$ channel. Two $\tau$-leptons are required to be opposite-sign and nearly back-to-back in the transverse plane. The analysis is also split into two categories, the $b$-veto and the $b$-tag categories, depending on the presence of a b-tagged jet. Figure 2 shows the signal acceptance times efficiency as a function of the mass of a neutral MSSM Higgs boson.

The dominant background is composed by events in which a jet is mis-reconstructed as a $\tau$ lepton, so called "fakes". A data-driven method is used to estimate fakes. Other backgrounds, like $Z+$ jet, diboson, $t \bar{t}$ and single top processes, are modeled by Monte Carlo simulation and corrected 


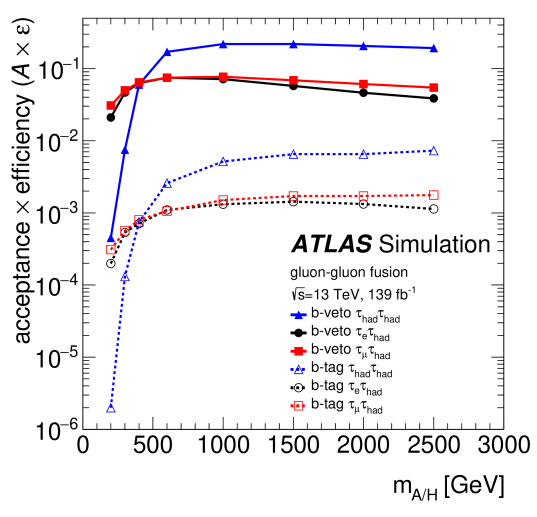

(a)

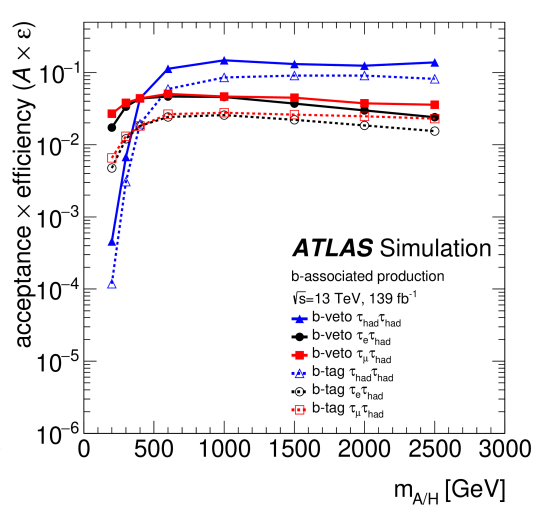

(b)

Figure 2: The signal acceptance times efficiency for (a) gluon-gluon fusion and (b) $b$-associated production of a neutral MSSM Higgs boson [2].

by data-driven method. Post-fit plots of the distribution of the total transverse mass $\left(m_{T}^{\text {tot }}\right)$ are shown as Figure 4.

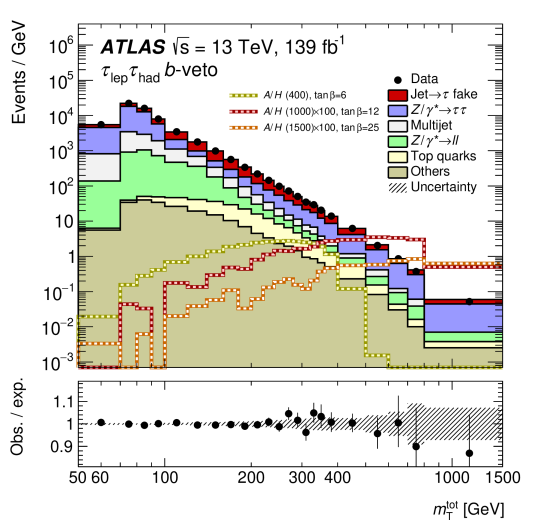

(a)

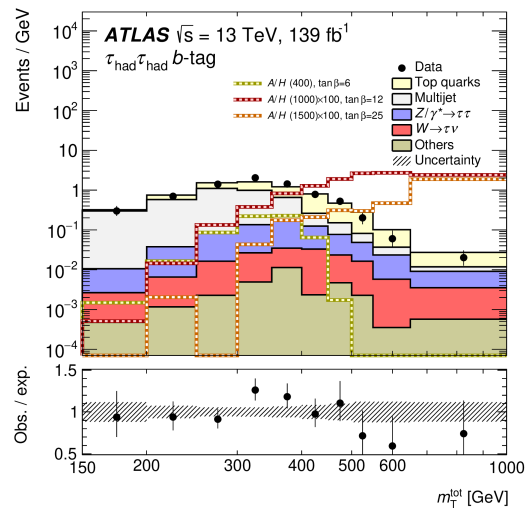

(b)

Figure 3: Post-fit plots of the distribution of the total transverse mass [2].

\section{Results}

A simultaneous fit of the $b$-veto and $b$-tag categories is performed on the distribution of the total transverse mass. No significant excess is observed in data. The data are in a good agreement with the SM predictions. 95\% confidence level (CL) upper limits for the production cross section times branching fraction of two production modes are calculated. Table 1 shows a summary table of the sources of systematic uncertainties considered in the analysis. Systematic uncertainties related to $\tau$ energy scale, $\tau$ identification efficiency, $Z+$ jet background modeling and the estimation of fake $\tau$ events are dominant uncertainties. 
Table 1: Relative increase in the expected 95\% C.L. upper limits for the production cross-section times branching fraction relative to the statistical only expected limit for each systematic uncertainty under consideration, shown for scalar bosons with mass of $400 \mathrm{GeV}$ and $1 \mathrm{TeV}$ [2].

\begin{tabular}{lrrrr}
\hline \hline NP name & ggF $(400 \mathrm{GeV})$ & $\mathrm{ggF}(1 \mathrm{TeV})$ & $\mathrm{bbH}(400 \mathrm{GeV})$ & $\mathrm{bbH}(1 \mathrm{TeV})$ \\
\hline Tau id. efficiency & 0.14 & 0.16 & 0.12 & 0.08 \\
Tau energy scale & 0.33 & 0.09 & 0.22 & 0.03 \\
Z+jets bkg. modeling & 0.27 & 0.19 & 0.08 & 0.04 \\
Mis-id. $\tau_{\text {had-vis }}$ bkg. & 0.22 & 0.01 & 0.14 & 0.03 \\
Others & 0.09 & 0.04 & 0.11 & 0.02 \\
\hline Total & 0.54 & 0.28 & 0.45 & 0.13 \\
\hline
\end{tabular}

Results are also interpreted in terms of several scenarios of the MSSM, as shown in Figure 4. In the $M_{h}^{125}$ scenario, where all superparticles are so heavy that they affect the production and decays of MSSM Higgs bosons, the data excludes $\tan \beta>8$ for $m_{A}=1.0 \mathrm{TeV}$ and $\tan \beta>21$ for $m_{A}=1.5$ $\mathrm{TeV}$ at the $95 \% \mathrm{CL}$.

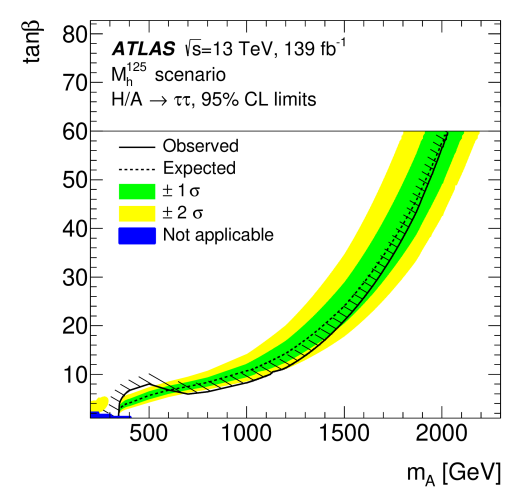

(a)

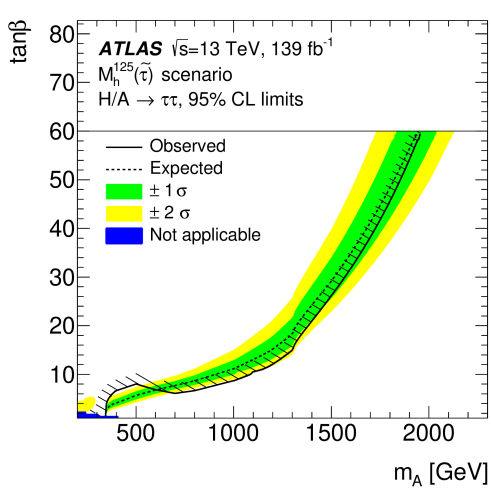

(b)

Figure 4: The $95 \%$ CL upper limits on $\tan \beta$ as a function of $m_{A}$ in (a) the $M_{h}^{125}$ scenario and (b) the $M_{h}^{125}(\tilde{\tau})$ scenario [2].

\section{References}

[1] ATLAS Collaboration, The ATLAS Experiment at the CERN Large Hadron Collider, JINST 3, S08003 (2008)

[2] ATLAS Collaboration, Search for heavy Higgs bosons decaying into two tau leptons with the ATLAS detector using pp collisions at $\sqrt{s}=13 \mathrm{TeV}$, Phys. Rev. Lett. 125, 051801[hep-ex/2002 . 12223] 